\title{
Determination of the thermal conductivity and specific heat capacity of neem seeds by inverse problem method
}

\author{
A.O. Kuye ${ }^{1, *}$, C.O.C. Oko ${ }^{2}$ and S.N. Nnamchi ${ }^{2}$ \\ ${ }^{1}$ Department of Chemical Engineering, University of Port Harcourt, PMB 5323, Port Harcourt, Nigeria. \\ ${ }^{2}$ Department of Mechanical Engineering University of Port Harcourt, PMB 5323, Port Harcourt, Nigeria.
}

Received 16 December 2008; Revised 14 July 2009; Accepted 2 December 2009

\begin{abstract}
Determination of the thermal conductivity and the specific heat capacity of neem seeds (Azadirachta indica A. Juss) using the inverse method is the main subject of this work. One-dimensional formulation of heat conduction problem in a sphere was used. Finite difference method was adopted for the solution of the heat conduction problem. The thermal conductivity and the specific heat capacity were determined by least square method in conjunction with Levenberg-Marquardt algorithm. The results obtained compare favourably with those obtained experimentally. These results are useful in the analysis of neem seeds drying and leaching processes.
\end{abstract}

Keywords: Neem seeds, thermophysical properties, thermal conductivity, specific heat capacity and inverse problem.

\section{Introduction}

Neem seeds (Azadirachta indica A. Juss) are derived from neem tree, which is endowed with insecticides and pesticides. The constituents are removed by leaching processes and the solvents recovered by drying process in a dryer. The drying of neem seeds in the fluidised bed dryer has been modelled with a lumped model [1] although the model results did not adequately represent the experimental results. By using a modified lumped model improved results were obtained [2]. However a distributed is expected to produce a more exact solution to the problem. But the application of the distributed model requires the prior knowledge of the thermal conductivity and the specific heat capacity of neem seeds.

Several methods exist in literature for estimation of thermophysical properties of materials [3, 4, 5]. Experimental determination of the thermal conductivity of a material could be through hot ball, hot wire, hot strip, pulse transient, stepwise transient, hot plate transient, hot disc transient or Gutaffson probe method. The calorimetric method is usually employed in the measurement of heat capacity. However, these experimental methods are not applicable wherever very accurate measurements are impossible due to extreme surface conditions, material geometry and size, etc. In such a situation, inverse problem method is most suitable because it uses the knowledge of the transient temperature field of the solid in conjunction with the heat conduction equation and LevenbergMarquardt algorithm to determine the thermophysical properties

* E-mail address: ayokuye@yahoo.com

ISSN: 1791-2377 (C) 2010 Kavala Institute of Technology. All rights reserved. more accurately [6].

The inverse heat conduction problem (IHCP) is concerned with the determination of the thermophysical properties of the medium, the initial or/and boundary temperature distribution for the given temperature field of the unsteady heat conduction.

It has been used in the determination of: high surface temperature where sensors could not be applied directly; thermophysical properties; temperature in internal combustion engines [7]; interface conductance between periodically contacting surfaces [8]; bulk radiation properties such as absorption or scattering coefficients [9]; and wall heat flux in a forced convection inside ducts $[6,10]$.

Therefore, this paper is aimed at estimating the two important thermophysical properties of neem seeds, the thermal conductivity and specific heat capacity using the IHCP approach, and to validate results by comparing them with those experimentally generated.

\section{Mathematical Formulation}

The formulation of inverse heat conduction problem originates from the direct heat conduction formulation. For neem seeds it is represented in spherical coordinate. The temperature distribution in the seeds is assumed to be radially dependent. Thus, for onedimensional unsteady state heat conduction equation in a spherical coordinate, the direct formulations are as follows: 
$\frac{\partial^{2} T}{\partial r^{2}}+\frac{2}{r} \frac{\partial T}{\partial r}+\frac{1}{k} g(r)=\frac{\rho c_{p}}{k} \frac{\partial T}{\partial t} \quad 0<r<R, t>0$

$k \frac{\partial T}{\partial r}=f_{1}(t)$ at $r=0, t>0$

$k \frac{\partial T}{\partial r}=f_{2}(t)$ at $r=R, t>0$

$T(r, 0)=F(r)$ for $t=0$ in $0 \leq r \leq R$

where $f_{1}(t), f_{2}(t), F(r), \rho c_{p}$ and $\mathrm{k}$ are all considered known.

The direct problem, Equation (1) is concerned with the determination of temperature distribution $T(r, t)$ in the interior region of the solid (neem seeds) as a function of time and position.

The inverse problem arises if any of the parameters is not known especially $\rho c_{p}$ and $\mathrm{k}$, but the transient temperature, boundary and initial conditions are known. Thus, the inverse formulation of Equations (1a-d) is as follows:

$\frac{\partial^{2} T}{\partial r^{2}}+\frac{2}{r} \frac{\partial T}{\partial r}+\frac{1}{k} g(r)=\frac{\rho c_{p}}{k} \frac{\partial T}{\partial t} \quad 0<r<R, \quad t>0$

$k \frac{\partial T}{\partial r}=0$ at $r=0, \quad t>0$

$k \frac{\partial T}{\partial r}+h_{b} T=h_{b} T_{g} \quad$ at $r=R, t>0$

$T(r, 0)=T_{s 0} \quad$ for $t=0$ in $0 \leq r \leq R$

Discretizing Equations $(2 \mathrm{a}, 2 \mathrm{~b}$ and $2 \mathrm{c}$ ) by method of finite difference $[6,11]$ for $g(r)=0$ give

$$
\begin{aligned}
& \frac{T_{i-1}^{n}-2 T_{i}^{n}+T_{i+1}^{n}}{(\Delta r)^{2}}+\frac{2}{i(\Delta r)} \frac{T_{i+1}^{n}-T_{i-1}^{n}}{2(\Delta r)}= \\
& =\frac{\rho c_{p}}{k} \frac{T_{i}^{n+1}-T_{i}^{n}}{\Delta t} ; i=1,2, \ldots, m-1 \\
& \left.\frac{\partial T}{\partial r}\right|_{r=R}=\frac{T_{-1}-T_{1}}{2(\Delta r)}=0, \quad \Rightarrow \\
& \Rightarrow T_{-1}=T_{1}, \quad \text { also, }, T_{-1}^{n}=T_{1}^{n}
\end{aligned}
$$

where $T_{-I}$ is a fictitious temperature; and

$$
\begin{aligned}
& k \frac{T_{m+1}-T_{m-1}}{2(\Delta r)}+h_{b} T_{m}=h_{b} T_{g}, \\
& \text { also, } k \frac{T_{m+1}^{n}-T_{m-1}^{n}}{2(\Delta r)}+h_{b} T_{m}^{n}=h_{b} T_{g}
\end{aligned}
$$

where $T_{m+1}$ is a fictitious temperature beyond the surface of the material. To obtain future temperature at the center of the neem seeds; the partial differential equation (Equation 2a) is replaced by a hyperbolic one by application of L'Hospital's rule to the second term in Equation (2a) in order to simplify the partial differential equation [6],

$$
\left.\frac{1}{r} \frac{\partial T}{\partial r}\right|_{r=0}=\left.\frac{(\partial / \partial r)\left(\frac{\partial T}{\partial r}\right)}{(\partial / \partial r)(r)}\right|_{r=0}=\left.\frac{\partial^{2} T}{\partial r^{2}}\right|_{r=0}
$$

Substituting Equation (4) in (2a), in the absence of the heat generation term, $g(r)=0$ gives:

$$
3 \frac{\partial^{2} T}{\partial r^{2}}=\frac{\rho c_{p}}{k} \frac{\partial T}{\partial t} \quad r \rightarrow 0, t>0
$$

Discretizing Equation (5a) gives

$$
3 \frac{T_{i-1}^{n}-2 T_{i}^{n}+T_{i+1}^{n}}{(\Delta r)^{2}}=\frac{\rho c_{p}}{k(\Delta t)}\left(T_{i}^{n+1}-T_{i}^{n}\right) ; i=0
$$

Setting $i=0$ in Equation ( $5 \mathrm{~b}$ ) gives,

$$
3 \frac{T_{-1}^{n}-2 T_{0}^{n}+T_{1}^{n}}{(\Delta r)^{2}}=\frac{\rho c_{p}}{k(\Delta t)}\left(T_{0}^{n+1}-T_{0}^{n}\right) ; \quad i=0
$$

Substituting $T_{-I}$ as defined in Equation (3b) into Equation (5c) gives,

$$
6\left(T_{1}^{n}-T_{0}^{n}\right)=\frac{\rho c_{p}}{k} \frac{(\Delta r)^{2}}{(\Delta t)}\left(T_{0}^{n+1}-T_{0}^{n}\right) ; i=0
$$

Rearranging Equation (3a) gives

$$
\begin{aligned}
& \left(1-\frac{1}{i}\right) T_{i-1}^{n}-2 T_{i}^{n}+\left(1+\frac{1}{i}\right) T_{i+1}^{n}= \\
& =\frac{\rho c_{p}}{k} \frac{(\Delta r)^{2}}{\Delta t}\left(T_{i}^{n+1}-T_{i}^{n}\right) ; \quad i=1,2, \ldots, m-1
\end{aligned}
$$

Setting $i=m$ in Equation (7) and substituting for $T_{m+l}$ as defined in Equation (3c) into Equation (7) gives,

$$
\begin{aligned}
& T_{m-1}^{n}-\beta_{m} T_{m}^{n}+\gamma_{m} T_{g}= \\
& =\eta_{m}\left(T_{m}^{n+1}-T_{m}^{n}\right)[K] ; i=m
\end{aligned}
$$

where

$$
\begin{aligned}
& \eta_{m}=\frac{1}{2 a} \frac{(\Delta r)^{2}}{\Delta t}[-] ; \quad a=\frac{k}{\rho c_{p}}\left[\mathrm{~m}^{2} / s\right] \\
& \gamma_{m}=\frac{h_{b}}{k} \frac{\Delta r}{m}(1+m)
\end{aligned}
$$




$$
\beta_{m}=\gamma_{m}+1
$$

Equations $(6,7$ and $8 \mathrm{a})$ are applied to compute future temperature distribution at the center, interior and the surface of the neem seeds, respectively.

For turbulent flow of air into the fluidised bed dryer, the heat transfer coefficient, $h_{b}$, is obtained from Nusselt $(\mathrm{Nu})$ dimensionless number,

$$
N u=p^{\prime} \operatorname{Re}^{r^{\prime}} \operatorname{Pr}^{0.33}=\frac{h_{b} d_{p}}{k_{\text {air }}}
$$

Where

$p^{\prime}=$ coefficient of Nusselt number and

$r^{\prime}=$ exponent of Nusselt number.

\section{Method of Solution}

The analytical solution of the inverse problem, Equation (2), for given temperature field is possible, say, by the use of the $\mathrm{Du}-$ hamel's or integral transform method. However, it is unstable even at small perturbation of input data [6]. Ozisik [6] has shown that the least square approach to the numerical solution of the problem, with appropriate choice of the minimisation procedure is unaffected by such instability.

$$
S(\hat{p})=\sum_{i=1}^{\Omega}\left[Y_{i}-\hat{T_{i}}\left(\hat{p}_{j}\right)\right]^{2}+\alpha^{*} \sum_{i=1}^{\Omega} \hat{p}^{2} \quad j=1,2, \cdots, m
$$

where $\alpha^{*}$ is known as the regularization parameter and must be in the range $0.0001<\alpha^{*}<0.1$. The regularization parameter could be negligible, as its value tends to lower limit. Thus, Equation (10a) reduces to

$$
S(\hat{p})=\sum_{i=1}^{\Omega}\left[Y_{i}-\hat{T}_{i}\left(\hat{p}_{j}\right)\right]^{2} \quad j=1,2, \cdots, m
$$

where $\hat{T}(\hat{p})$ is estimated ground neem seeds temperature, which is defined by Equations (6,7 and 8). Equation (10b), is minimized by differentiating it with respect to each of the unknown parameters $\hat{p}_{j}(j=1,2, \ldots m)$ and then setting the resulting expression equal to zero:

$$
\frac{\partial S}{\partial \hat{p}_{j}}=2 \sum_{i=1}^{\Omega}\left(\frac{\partial \hat{T}_{i}}{\partial \hat{p}_{j}}\right)\left[T_{i}(\hat{p})-Y_{i}\right]=0 \quad j=1,2, \cdots, m
$$

In matrix notation Equation (11a) becomes

$$
\frac{\partial S}{\partial \hat{p}}=2 J^{T}(\hat{T}-Y)=0
$$

where $J=$ Jacobian defined as.

$$
J=\frac{\partial T}{\partial \hat{p}}=\left[\begin{array}{cccc}
\frac{\partial \hat{T}_{1}}{\partial \hat{p}_{1}} & \frac{\partial \hat{T}_{1}}{\partial \hat{p}_{2}} & \cdots & \frac{\partial \hat{T}_{1}}{\partial \hat{p}_{m}} \\
\frac{\partial \hat{T}_{2}}{\partial \hat{p}_{1}} & \frac{\partial \hat{T}_{2}}{\partial \hat{p}_{2}} & \cdots & \frac{\partial \hat{T}_{2}}{\partial \hat{p}_{m}} \\
\vdots & \vdots & \ddots & \vdots \\
\frac{\partial \hat{T}_{\Omega}}{\partial \hat{p}_{1}} & \frac{\partial \hat{T}_{\Omega}}{\partial \hat{p}_{2}} & \cdots & \frac{\partial \hat{T}_{\Omega}}{\partial \hat{p}_{m}}
\end{array}\right]
$$

where

$\hat{T}=\left[\begin{array}{c}\hat{T}_{1} \\ \hat{T}_{2} \\ \vdots \\ \hat{T}_{\Omega}\end{array}\right], \quad Y=\left[\begin{array}{c}Y_{1} \\ Y_{2} \\ \vdots \\ Y_{\Omega}\end{array}\right]$,

$$
\hat{p}=\left[\begin{array}{c}
\hat{p}_{1} \\
\hat{p}_{2} \\
\vdots \\
\hat{p}_{m}
\end{array}\right], \Omega=(m+1)(n+1)
$$

According to Levenberg-Marquardt [6], the estimated parameters are expressed as

$\hat{p}^{l+1}=\hat{p}^{l}-\left(J^{T} J+\mu_{l} I\right)^{-1} J^{T}(Y-T), \quad l=1,2,3, \cdots$

where

$\hat{p}=\{k, c\}^{T} ; \mathrm{k}$ and $\mathrm{c}$ are the thermal conductivity and specific heat capacity, respectively;

$\mu_{l}$ is the damping parameter (arbitrarily fixed).

The elements of the Jacobian are obtained by perturbing the parameters (thermal conductivity and heat capacity). The perturbation is represented as

$$
\begin{aligned}
& \frac{\partial T}{\partial k} \approx \frac{T(k+\Delta k, c ; t)-T(k, c ; t)}{\Delta k} \\
& \frac{\partial T}{\partial c} \approx \frac{T(k, c+\Delta c ; t)-T(k, c ; t)}{\Delta c}
\end{aligned}
$$

Then Levenberg-Marquardt algorithm converges as

$$
\left|\hat{p}^{l+1}-\hat{p}^{l}\right| \leq \varepsilon
$$

Fortran77 programme has been developed which implements Levenberg-Marquardt algorithm for the determination of neem seeds thermophysical properties.

Moreover, as away of rough comparison, the same data on the thermal conductivity and specific heat capacity of neem seeds were generated experimentally by calometric and hot wire methods, respectively $[3,4,5]$. 


\section{Results and Discussion}

Tables 1 and 2 contain the input data for neem seeds in the fluidised bed dryer, transient temperature and the physical properties, respectively. Table 3 contains the input data for Nusselt equation used in computing the heat transfer coefficient for turbulent flow. All the input data were used to obtain the numerical solution of the inverse problem. Figures $(1-3)$ show the experimental and the estimated neem seeds temperatures. The estimated neem seeds temperatures were obtained by the numerical solution of Equation (2) which describe the heat transfer process in the fluidised bed dryer. The deviations between the estimated neem seeds temperatures and the experimental data were minimised by using Levenberg-Marquardt optimisation scheme. The neem seeds temperatures differ from those of the fluidising air temperatures, which were kept constant. Figures (1 - 3) show that neem seeds temperatures were building with time and further approached the air temperature after 4000 [sec].

Table 1. Solid Transient Temperature (Input Data for IHCP Estimation)

\begin{tabular}{cccc} 
& \multicolumn{3}{c}{ Heating air temperature $\left({ }^{\circ} \mathbf{C}\right)$} \\
\cline { 2 - 4 } (second) & $\mathbf{4 0}$ & $\mathbf{6 0}$ & $\mathbf{8 0}$ \\
\cline { 2 - 4 } 0 & 287.50 & 291.00 & 293.80 \\
200 & 286.36 & 291.49 & 303.35 \\
400 & 288.05 & 289.25 & 300.04 \\
600 & 287.14 & 289.96 & 302.55 \\
800 & 283.36 & 292.02 & 305.70 \\
1000 & 286.25 & 292.45 & 317.73 \\
1200 & 289.30 & 294.58 & 328.61 \\
1400 & 292.29 & 297.83 & 336.00 \\
1600 & 295.09 & 300.84 & 339.89 \\
1800 & 297.65 & 309.39 & 344.24 \\
2000 & 300.50 & 315.88 & 346.72 \\
2200 & 302.00 & 319.21 & 348.70 \\
2400 & 303.78 & 320.87 & 349.59 \\
2600 & 307.22 & 323.43 & 350.42 \\
2800 & 309.01 & 325.65 & 351.31 \\
3000 & 310.73 & 327.40 & 351.95 \\
3200 & 311.94 & 328.36 & 352.20 \\
3400 & 312.71 & 329.17 & 353.51 \\
3600 & 313.17 & 330.17 & 352.93 \\
3800 & 313.42 & 330.45 & 353.05 \\
4000 & 313.42 & 333.45 & 353.31 \\
\hline
\end{tabular}

Source: Espinosa et al [1].

Table 2. Physical Properties of Neem seeds (input data)

\begin{tabular}{lcc}
\hline \multicolumn{1}{c}{ Parameter } & Ground * & Unground \\
\hline Weight mean diameter, $\times 10^{-3}[\mathrm{~m}]$ & 0.96 & 8.8 \\
Average density, $\left[\mathrm{kg} / \mathrm{m}^{3}\right]$ & 690 & 690 \\
Exposed area per dry mass, $\left[\mathrm{m}^{2} / \mathrm{kg}\right]$ & 9.11 & 0.92 \\
\hline
\end{tabular}

* Source: Espinosa et al [1].

Table 3. Nusselt constants (input data)

\begin{tabular}{ccc}
\hline $\begin{array}{c}\text { Air temperature, } \\
\mathbf{T}_{\mathbf{g}}\left[{ }^{\circ} \mathbf{C}\right]\end{array}$ & $\begin{array}{c}\text { Coefficient, } \\
\boldsymbol{p}^{\prime}\end{array}$ & $\begin{array}{c}\text { Exponent, } \\
\boldsymbol{r}^{\prime}\end{array}$ \\
\hline 40 & 0.0247272 & 0.13000 \\
60 & 0.0266450 & 0.13000 \\
80 & 0.0285157 & 0.13000 \\
\hline
\end{tabular}

\footnotetext{
* Source: Nnamchi [12].
}

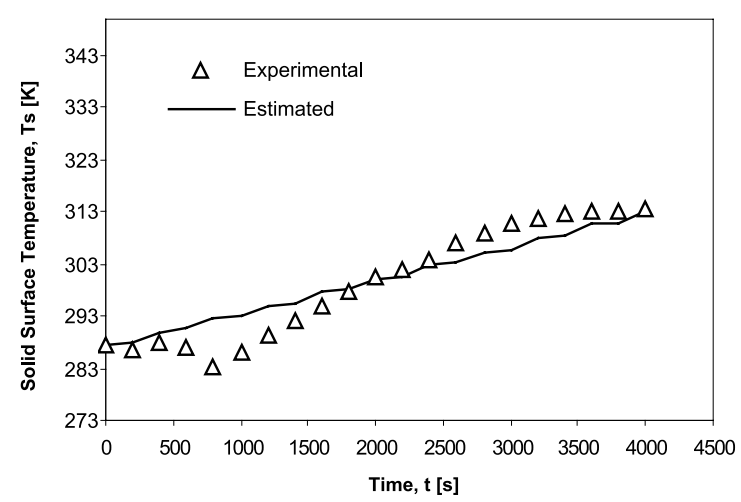

Figure 1. Solid Surface Temperature for Heating Air at 313.15 [K].

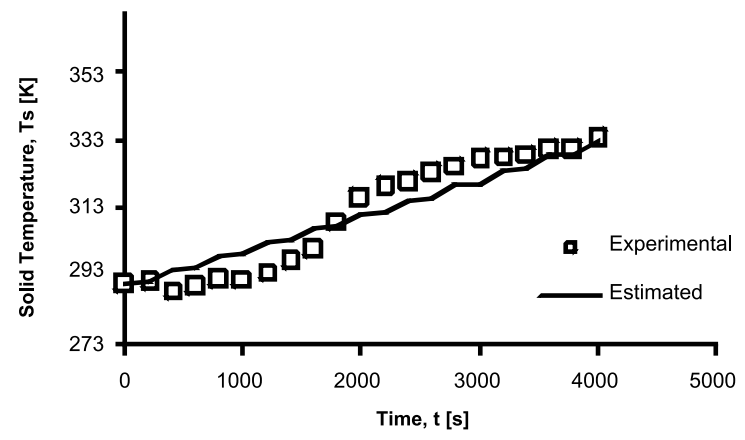

Figure 2. Solid Temperature for Heating Air at 333 [K].

Figures (4 and 5) show the results of the thermophysical properties: the thermal conductivity and the specific heat capacity respectively. The two graphs show that the thermophysical properties are linearly dependent on the temperature. Essentially, Equations (1 and 2) show that the thermal conductivity and specific heat capacity of neem seeds are constant, since they are not varying with space. Rather they are function of neem seeds temperature. Hence, at mean solid temperature of 311 [K], the thermal conductivity and specific heat capacity of neem seeds by IHCP method is $0.023186[\mathrm{~W} / \mathrm{mK}]$ and $1488.47[\mathrm{~J} / \mathrm{kgK}]$ respectively.

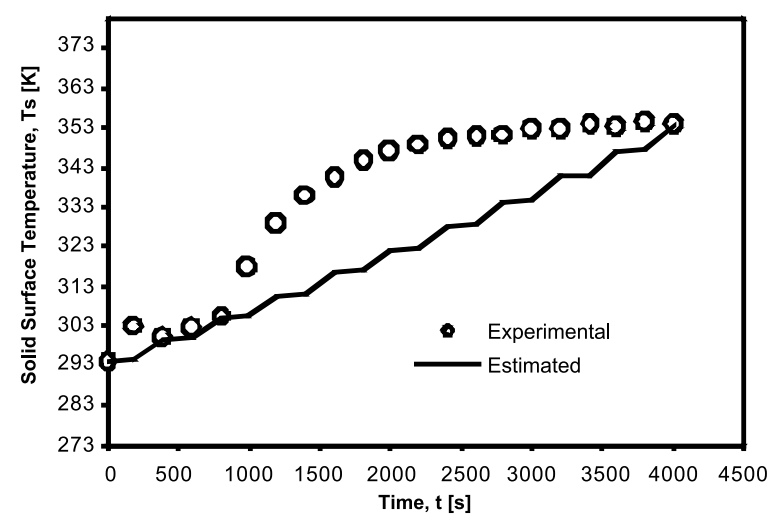

Figure 3. Solid Surface Temperature for Heating Air at 353.15 [K]. 


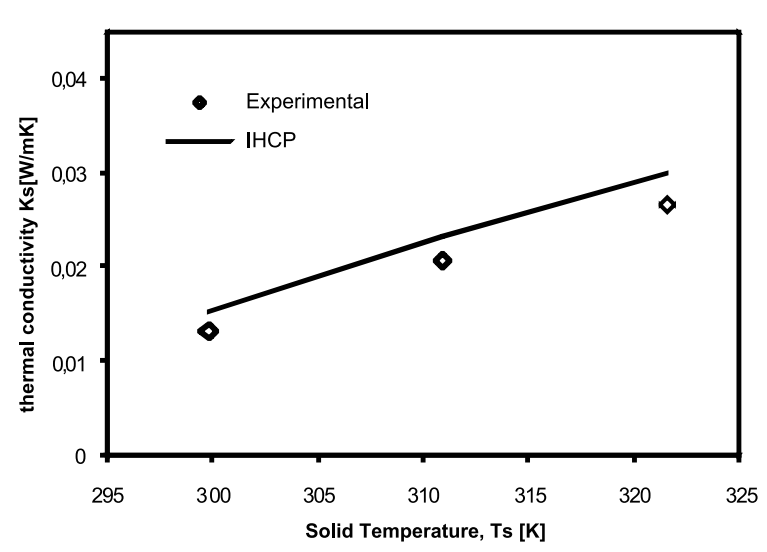

Figure 4. Comparison of Thermal Conductivity of Neem Seeds.

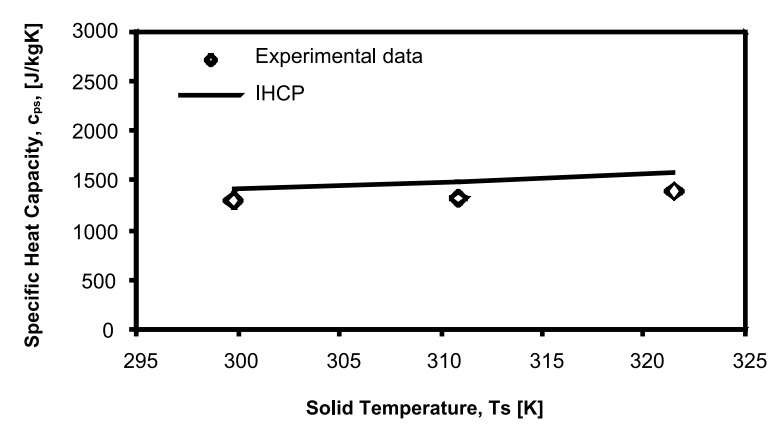

Figure 5. Comparison of Specific Heat Capacity of Neem Seeds.
Also, at mean solid temperature of $311[\mathrm{~K}]$, the thermal conductivity and specific heat capacity of neem seeds by experimental method is $0.020268[\mathrm{~W} / \mathrm{mK}]$ and $1329.62[\mathrm{~J} / \mathrm{kgK}]$ respectively.

Moreover, Figures (4 and 5) show that the IHCP results agreed with those of experimental results. Thus, these results will be useful in application of distributed model in modelling the drying and leaching processes for an improved model results.

\section{Conclusion}

The temperatures of neem seeds have been estimated using the numerical solution of the thermal diffusion equation, the results approximated those of the measured neem seeds temperatures. The thermophysical properties of neem seeds (the thermal conductivity and the specific heat capacity) have been determined numerically by IHCP method. The thermophysical properties are in good agreement with the experimental results. Hence, IHCP is reliable method of determining thermophysical properties solids where very accurate measurements are impossible.

\section{References}

1. Espinosa, R., Lagerstedt, J., Nyman, T. and Martinez, J. (2002), Drying of Ground Neem seeds in a Fluidised Bed Dryer, Proceedings of the 13th International Drying Symposium (IDS' 2002), Beijing, China, vol. B, 1360.

2. Kuye, A; Oko, C.O.C. and Nnamchi, S.N. (2007). Simulation of the Drying Characteristics of Ground Neem seeds in a Fluidised Bed. J. of Eng. Sc. and Tech., Vol. 2, No. 1, 21-31.

3. Alifanov, O.M. and Kilbanov, M.V. (1985). J. Eng. Phys. 48, 730-735.

4. Artyuklin, E.A. and Özişik, M.N. (1983). J. Eng. Phys. 45, 1275-1281.

5. Hunag, C.H. and Özişik, M.N. (1990). Int. J. Heat and Fluid Flow 11, 262 -268 .

6. Özişik, M.N. (1993). Heat Conduction, 2nd ed. John Wiley, New York, 571-616.
7. Alkidas, A.L.J. (1980). Heat Transfer. 102, 189-193.

8. Flach, G.P. and Ozisik, M.N. (1988). J. Heat Transfer, 110, 821-829.

9. Ho, C.H. and Özişik, M.N. (1989). Int. J. Heat Mass Transfer, 32, 335341.

10. Hensel, E. (1991). Inverse Theory and Application for Engineers, Prentice Hall, Englewood Cliffs N. J.

11. Chapra, S.C. and Canale, R.P. (1998). Numerical Methods for Engineers: with Programming and Software, 3rd ed., McGraw-Hill, Boston, 812847.

12. Nnamchi, S.N. (2003). Development of predictive drying model for ground neem seeds, M.ENG Thesis, University of Port Harcourt, Nigeria.

\section{Nomenclature}

$$
\begin{aligned}
& \mathrm{A}_{\mathrm{s}} \\
& \mathrm{C}_{\mathrm{s}} \\
& \mathrm{c}_{\mathrm{ps}} \\
& \mathrm{d} \\
& g \\
& \mathrm{~h}_{\mathrm{b}} \\
& \mathrm{k} \\
& \mathrm{m}_{\mathrm{s}} \\
& \hat{p} \\
& \mathrm{r}, \mathrm{R}
\end{aligned}
$$

total surface area of neem seeds volumetric heat capacity of neem seeds specific heat capacity of neem seeds diameter of neem seeds heat generation term heat transfer coefficient thermal conductivity of neem seeds mass of solid Parameter $\left(k\right.$ or $\left.c_{p}\right)$ radius of neem seeds $\mathrm{m}^{2}$

$\mathrm{kJ} / \mathrm{m}^{3} \mathrm{~K}$ $\mathrm{J} / \mathrm{kgK}$ $\mathrm{m}$

$\mathrm{W} / \mathrm{m}^{3}$

$\mathrm{W} / \mathrm{m}^{2} \mathrm{~K}$

$\mathrm{W} / \mathrm{mK}$

$\mathrm{kg}$

$\mathrm{m}$ 
$\mathrm{S}$

$\mathrm{T}_{\mathrm{g}}$

$\mathrm{T}_{\mathrm{s}}$

$\mathrm{t}$

Y

\section{Greek letters}

$\Delta$

$\varepsilon$

$\mu_{l}$

$\alpha^{*}$

$\alpha$

\section{Subscripts}

0

b

e

g

i

in

m

o

\section{Superscripts}

l

0

n

$\mathrm{T}$ sum of squares

temperature of gas (air)

$\mathrm{K}^{2}$

temperature of solid

$\mathrm{K}$

time

K

measured temperature at surface

change in parameter

convergence limit

damping parameter

regularization parameter

thermal diffusivity

$\mathrm{m}^{2} / \mathrm{s}$

initial value

interface

equilibrium

air

iterations for time

inside

space

outside

iteration values

superficial, initial condition

time

transpose 